\title{
ELECTROSTATIC STRESS ANALYSIS OF HIGH VOLTAGE CABLES
}

\author{
Khanjan N. Jariwala ${ }^{1}$ \\ ${ }^{I}$ Department of Electrical Engineering, Charotar University of science and Technology, Changa, Gujarat, India
}

\begin{abstract}
Power cable as one of the main components of power networks, play an important role in electric power transmission between different zones. In cable, stress will maximum nearer to the surface of the conductor and it will get decreases as we move away from core to outer sheath. So, dielectric will be unnecessarily strong and expensive. Moreover, in cable insulation system, due to presence of pollutant such as air voids, water voids and also occurrence of water treeing phenomena, local field increases. As a result, enhancing electric field may cause to local breakdown in cable insulation. The presence of water within the insulation material can only lead to forming water trees if a sufficient electric field exists which may shorten life of cable. In this thesis, Finite element method is used to computing and evaluating of the electric field in the power cable by considering to existence of water particle and air void inside the polyethylene insulation. For equal stress distribution, grading methods are also considered. For all this purpose FEMM software is used for modeling. The results indicate that the stress is influenced by the void permittivity, shape of void, sharpness of void and also void position. By providing grading of the cable we can equally distribute the stress along the cable insulation.
\end{abstract}

Keywords - Cable grading, FEMM, Finite element method, cavity

\section{INTRODUCTION}

Cables are one of the important equipment used in power networks and industrial factories. High voltage cables are used for transmission and distribution of electric power. Cables are generally used for underground application. In present scenario, XLPE is used increasingly as an insulating material because of its more advantageous over other materials. The insulation of cable has to continuous withstood electrical, thermal as well as mechanical stress. If this stress exceeds its limit, it may damage the cable. Electric insulation is main part of power cable and its quality affects the expected life [1]. In addition to this issue, other factor such as partial discharge, impurity, roughness, cavities and space charge will affect the insulation life time [2-4]. Critical field of Paschen is calculated [3]. The electrical stress is highest at surface of the core and it is highest at inner radius of the sheath. So, there will be large difference between maximum and minimum stress in insulation. So, as a result we can say that dielectric material will not fully utilize. For uniform stress distribution, grading of cable is used. One of the major problems, which shorten the life of high voltage cable, is presence of water or void or cavity inside the insulation material of the cable. By the presence of such pollutant, stress in the insulation increase very much at that particular point and may get leads to breakdown of the insulation. So analysis of this stress in this insulation is necessary to get idea about the life of the cable. Stress analysis using the defect like water bubble or void or cavity is carried out in this work.

\section{ANALYTICAL METHOD}

One of the most important problems in high voltage engineering is electric field calculation which requires solution of Laplace and Poisson equations. In Past studies, electric field is introduced as a source of corona around conductor imperfect partial discharge ad early insulation fatigue. In order to study the effect of cavity inside insulation of cable spherical cavity with radius $r_{c}$ radius is considered inside insulation with $R$ radius. Internal and External electric field of cylindrical cavity is calculated via following equation (1-2),

$$
E(\mathrm{r})=E_{0}\left(i_{r}\left[1+\frac{\left(\varepsilon_{r}-\varepsilon_{0}\right)}{\varepsilon_{r}-\varepsilon_{0}} \cdot \frac{r_{c}^{2}}{r^{2}}\right]\right) \cos \theta-\left(i_{\theta}\left[1-\frac{\left(\varepsilon_{r}-\varepsilon_{0}\right)}{\varepsilon_{r}-\varepsilon_{0}} \cdot \frac{r_{c}^{2}}{r^{2}}\right]\right) \sin \theta
$$

for $r>r_{c \ldots}(1)$

$$
E(\mathrm{r})=\mathrm{E}_{0}\left(\frac{2 \varepsilon_{0}}{\varepsilon_{r}+\varepsilon_{0}}\right)\left(i_{r} \cos \theta-i_{\theta} \sin \theta\right) \text { for } 0<\mathrm{r}<\mathrm{rc} .
$$

In these equations (1-2), cavity surface conductivity as a factor affecting on electric field intensity, is neglected. But most of the time non linear environment and problem geometry are so complicated that analytical solution is so hard. Due to this reason, researches tried to find new calculating methods to obtain electric field. FEM has increasingly developed in this context. Electric field equations solution by this method is based on Maxwell equations with boundary conditions. 
In practice, cavities have irregular shapes and thus analytical solutions for such non-ideal voids are impossible. But numerical solutions can be obtained by solving Maxwell's equation with appropriate boundary conditions.

\section{NUMERIC METHOD-DETERMINING \\ ELECTRIC FIELD INSIDE CABLE UNDER VARIOUS CONDITION OF CAVITY PRESENCE}

Due to singularity point at the corner of a cavity, the analytical method can not be used without simplifying assumptions [5]. In this section, we represent different conditions of cavity presence in single core cable as shown in fig (1) by using FEMM software to calculate electric field. High voltage cable cross section is as under,

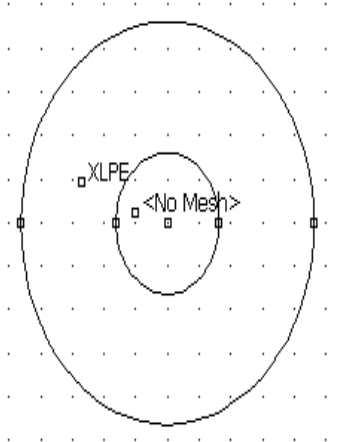

Fig 1 Cross section of cable under study

Other technical characteristics of the cable is shown in table-1,

Table 1: Cable characteristics under study

\begin{tabular}{|l|l|}
\hline Cable Characteristics & Parameters \\
\hline Rated Voltage & $33 \mathrm{KV}$ \\
\hline Number of Core & 1 \\
\hline Area of conductor & $400 \mathrm{~mm} 2$ \\
\hline Radius of conductor & $1.30 \mathrm{~cm}$ \\
\hline Overall diameter & $7 \mathrm{~cm}$ \\
\hline $\begin{array}{l}\text { Insulation } \\
\text { constant(XLPE) }\end{array}$ & 2.3 \\
\hline Water permittivity & 81 \\
\hline Air permittivity & 1 \\
\hline
\end{tabular}

\section{RESULTS AND DISCUSSION}

\subsection{Influence of Cavity Position on Electric Field Variation}

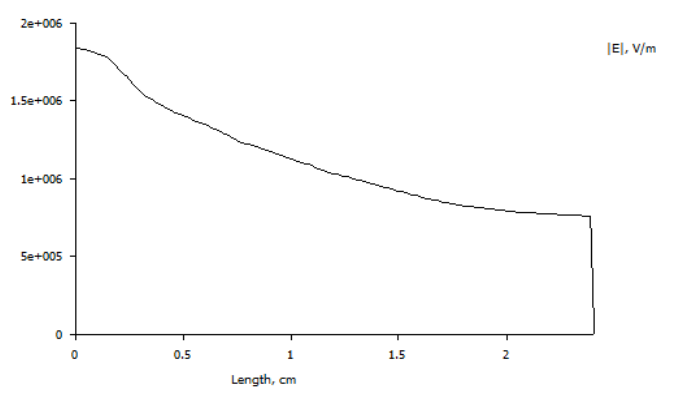

Fig 2: Electric field distribution without cavity

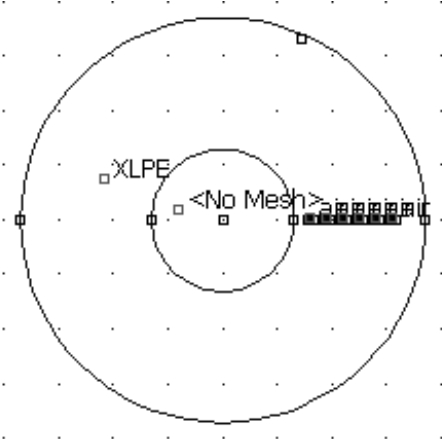

Fig 3: Cable with six cavities

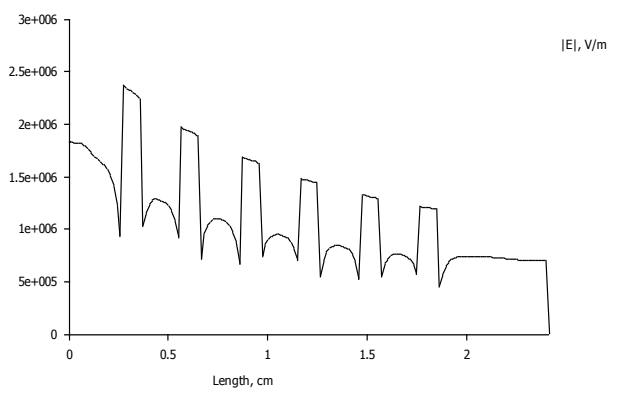

Fig 4: Field distribution in presence of cavity at different locations (Axially)

In this section, in order to study cavity position impact on cable electric field, we first simulate cable without any cavity. Then we will simulate for a case when 6 cavities having radius 0.05 $\mathrm{mm}$, are located radial inside cable insulation by equal distance. Fig-2 shows in absence of cavity, electric field decreases logarithmically along insulation section. Fig-3 shows that cavity near to the cable conductor has more electric field value in comparison to that of a cavity located near cable insulation surface. In this study we want to evaluate electric field value in 
side cable under various conditions and we neglect Partial discharge phenomena.

\subsection{Influence of the Shape of the Cavity}

In this section we want to study the effect of air cavity shape on electric field of the cable. It is supposed that geometric shape of cavity is cylindrical, spherical and elliptical and it is located inside cable insulation. Now if we convert 3-D model of a cavity shape in to 2-D model as a result cylindrical cavity would be rectangle, spherical cavity would be circle. We will do simulation for all three cavities.

Table 2: Maximum electrical stress for various cavity shapes

\begin{tabular}{|l|l|}
\hline Cavity Shape & $\begin{array}{l}\text { Maximum stress } \\
(\mathrm{V} / \mathrm{m})\end{array}$ \\
\hline Cylindrical & $2.75 \mathrm{e}+006$ \\
\hline Spherical & $2.45 \mathrm{e}+006$ \\
\hline Elliptical & $2.23 \mathrm{e}+006$ \\
\hline
\end{tabular}

From table -2, it infers that electric field enhancement is highest for cylindrical cavity than spherical or elliptical cavity. So, it is more likely to initiation partial discharge in this case.

\subsection{Influence of Dielectric Medium}

In this section we will study effect of permittivity of material in electric field variation. For that two kinds of cavities will be considered.

Fig-5 shows electric field distribution when air cavity occurs at the middle of the cable. It is inferred that in case of air cavity, electric field will drastically enhanced. It is due to permittivity difference. Same as this, electric field is also enhanced as shown in fig- 6 in case for cavity having water partials at the middle.

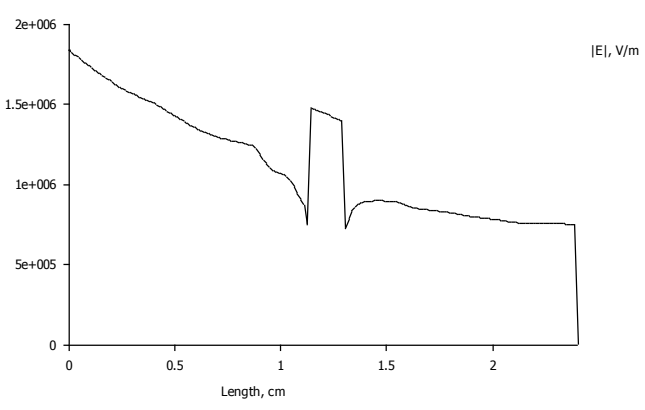

Fig 5: Electric field distribution with water cavity (permittivity=1)

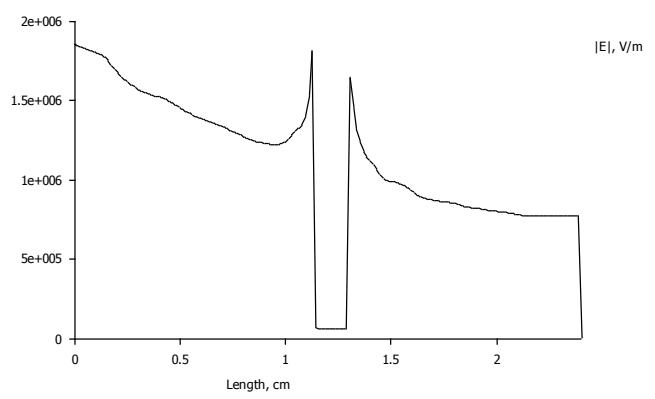

Fig 6: Cable with water cavity (permittivity $=81$ )

Fig-5 and Fig-6 shows that Electric field inside water cavity is decreased drastically because water permittivity coefficient is 33 times more than that of cable insulation.

\subsection{Influence of Sharpness of Cavity}

In this section we will see the effect of sharpness of cavity. For that we will consider elliptical cavity and we will change its sharpness.

Table 3: Max field stress for different arc angles

\begin{tabular}{|l|l|}
\hline Arc Angle & Maximum Field stress $(\mathrm{V} / \mathrm{m})$ \\
\hline For 100degree & $2.0 \mathrm{e}+006$ \\
\hline For 120degree & $2.25 \mathrm{e}+006$ \\
\hline For 130 degree & $2.33 \mathrm{e}+006$ \\
\hline For 140 degree & $2.49 \mathrm{e}+006$ \\
\hline
\end{tabular}

Results from upper table-3 infer that as we increase arc angle from 100 to 140 degrees, electric field enhancement is increases accordingly.

\section{CONCLUSIONS}

Analytical method can calculate the mean stress in the void. However, the field distribution in the vicinity of a void of nonstandard shape is difficult to calculate by analytical method. In this study, the results show the important influence of shape of void on electric stress within it. As it well Known, the stress in the void cavity is enhanced and this is an important consideration for PD activity. The cavity stress enhancement increase as permittivity of dielectric increase. Stress enhancement is grater for cavity nearer to the surface of the conductor. Moreover, electric field inside void is also function of its edge sharpness. As its sharpness increases, electric field enhancement is also increases. Also results show that electric field is a function of cavity shape. According to result obtained, electric field inside cylindrical cavity is higher than that of spherical one and in spherical cavity is higher than elliptical one. 


\section{REFERENCES}

[1]. H.N.O.T. Blackburn, B.T. Phung, H. Zhang and R.H. Khwaja, , The university New south Wales, Australia, "Investigation of Electric Field Distribution in power cable with voids", IEEE transactions, 2006

[2]. H. A. Illias, Q. L. Ng, A. H. A. Bakar, H. Mokhlis, UMPEDAC Electrical Engineering Department Faculty of Engineering, University of Malaya Kuala Lumpur, Malaysia, and A. M. Ariffin, College of Engineering Universiti Tenaga Nasional Selangor, Malaysia, "Electric field distribution in $132 \mathrm{kV}$ XLPE cable termination model from finite element method", 2012 IEEE International Conference on Condition Monitoring and Diagnosis, 23-27 September 2012, Bali, Indonesia

[3]. T. Seghir1, D.Mahi1, T.Lebey2, D.Malek2,1Material laboratory, Electrical Engineering Institute, Amar Thelidji University of Laghouat, Algeria,2Electrical Engineering Laboratory, University of Paul Sabatier, Toulouse France, "Analysis of the Electric Field and Potential Distribution in Cavities Inside Solid Insulating Electrical Materials", Experts from the proceedings of the COMSOL user conference 2006 Paris.

[4]. Mohamed Alsharif, Peter A. Wallace, Donald M. Hepburn, Chenke ZHOU, Caledonian University-UK Glasgow, “ Partial Discharge Resulting From Internal Degradation in Underground MV cables: Modeling and Analysis", IEEE transactions, 2009

[5]. D.A. Hovath, PE and R.L. Steinmen, PhD Advent Engineering services, inc. Ann Arbor, MI, USA, “ Relationship of electric insulation void content with electric cable normalized capacitance", 2001 Annual report Conference on Electrical insulations and Dielectric Phenomena, IEEE Transaction, 2001

[7]. S. Lachini, A.Gholani, M.Mirzaie, Iran university of Science and Technology, "Determining Electric field distribution in high voltage cable in presence of cavity", UPEC, 31st Aug-3rd Sept, 2010

[8]. K. Rajagopala, K. Panduranga Vittal, Hemsingh Lunavath, TELKOMNIKA, "Computation of Electric field and thermal Properties of 3-phase cable", Volume 10,No.2, Issue 4, June 2012 p.p $265-274$

[9]. H.S.B Elayyan,M.H. Abderrazzaq, "Electric field Computation in wet cable insulation using finite element approach", IEEE transaction on Dielectrics and electrical insulation, Volume 12,No.6, December 2005, p.p 1125-1133

[10]. M. U. Zuberi, A. Masood, E. Husain and A. Anwar, "Estimation of Partial Discharge Inception Voltages Due to Voids in Solid Sheet Insulation", IEEE Conference on Electrical Insulation, Ottawa, Ontario, Canada, pp-124-128,2 to 5 June 2013 